\title{
Fault Detection Filter Design for Networked Multi-rate Systems with Fading Measurements and Randomly Occurring Faults
}

\author{
Yong Zhang, Zidong Wang, Lei Zou and Zhenxing Liu
}

\begin{abstract}
In this paper, the fault detection problem is investigated for a class of networked multi-rate systems (NMSs) with network-induced fading channels and randomly occurring faults. The stochastic characteristics of the fading measurements are governed by mutually independent random channel coefficients over the known interval $[0,1]$. By applying the lifting technique, the system model for the observer-based fault detection is established. With the aid of the stochastic analysis approach, sufficient conditions are established under which the stochastic stability of the error dynamics for the state estimation is guaranteed and the prescribed $H_{\infty}$ performance constraint on the error dynamics for the fault estimation is achieved. Based on the established conditions, the addressed fault detection problem of NMSs is recast as a convex optimization one that can be solved via the semi-definite program method, and the explicit expression of the desired fault detection filter is derived by means of the feasibility of certain matrix inequalities. The main results are specialized to the networked single-rate systems that are a special case of the NMSs. Finally, two simulation examples are utilized to illustrate the effectiveness of the proposed fault detection method.
\end{abstract}

\section{Index Terms}

Networked multi-rate systems; Fading measurements; Randomly occurring faults; Fault detection.

\section{INTRODUCTION}

In networked control systems (NCSs) [1], [2], in addition to the well-studied communication delays [3], [4], packet dropouts [5]-[8] and signal quantization [9]-[11], the channel fading phenomenon is often unavoidable due mainly to the multi-path propagation, shadowing effects from obstacles, as well as the path loss. Up to now, the stability and state estimation problems for the networked systems with fading measurements have drawn some initial research attention [12]-[17]. On the other hand, most available literature concerning NCSs has assumed the single-rate sampled-data setting for the underlying system. However, in practice, especially for large-scale networked systems, the elements of the control system may be structured distributively, that is, the sensors, actuators and controller are connected by communication networks. For such kind of NCSs, faster A/D and D/A conversions would lead to better performance but

This work was supported in part by the National Natural Science Foundation of China under Grants 61104027, 61174107 and 61329301, the Royal Society of the U.K., and the Alexander von Humboldt Foundation of Germany.

Y. Zhang and Z. Liu are with the School of Information Science and Engineering, Wuhan University of Science and Technology, Wuhan 430081, China. (Email: zhangyong77@wust.edu.cn)

Z. Wang is with the Department of Computer Science, Brunel University London, Uxbridge, Middlesex, UB8 3PH, United Kingdom. He is also with the Faculty of Engineering, King Abdulaziz University, Jeddah 21589, Saudi Arabia. (Email: Zidong.Wang@brunel.ac.uk)

L. Zou is with the Research Institute of Intelligent Control and Systems, Harbin Institute of Technology, Harbin 150001, China. 
also mean higher implementation cost. Allowing different speeds for A/D and D/A conversions results in satisfactory trade-offs between the performance and implementation cost. As such, the scheme of multi-rate sampled-data (MSD) arise naturally and has become a research focus for many years, see [18]-[22].

In order to meet the ever-increasing demand for higher performance, higher safety, and reliability standard, the fault detection problem has been an active research area for several decades [23], [24]. Recently, the fault detection (FD) problem of networked control systems [25]-[27], [29] has become a rather hot topic. For example, to deal with the FD problem of nonlinear networked systems, the T-S fuzzymodel-based fault detection problem has been studied in [30] for NCSs with Markov delays. In [31], an FD framework has been proposed for a class of nonlinear NCSs via a shared communication medium. On the other hand, the FD problem of MSD systems has been investigated in [18]-[20]. To date, the FD problem has not been adequately examined for networked multi-rate systems (NMSs), not to mention the cases when fading measurements and randomly occurring faults are simultaneously presented.

In this paper, we aim to investigate the fault detection problem for a class of NMSs with fading measurements and randomly occurring faults. Our main contributions can be highlighted as follows: (1) the system model is comprehensive that covers networked multi-rate sampled-data dynamics, fading measurements and randomly occurring faults, thereby better reflecting the reality; (2) by using the lifting technique, the FD problem for networked multi-rate sampled-data systems is investigated that caters for fading measurements and randomly occurring faults; and (3) the sufficient conditions are establish to quantify the relationships between the $H_{\infty}$ performance, the fault occurrence probability as well as the multiple of period $h$.

The rest of this paper is outlined as follows. In Section II, the multi-rate sampled-data system with network-induced randomly occurring faults and measurements fading is introduced. Section III uses lifting technique to establish the model for the multi-rate fault detection dynamics. In Section IV, by employing the Lyapunov stability theory, some sufficient conditions are established in the form of matrix inequalities, and then the fault detection gain is obtained by solving a convex optimization problem. Two illustrative examples are given in Section V to demonstrate the effectiveness of the results obtained. Finally, conclusions are drawn in Section VI.

Notation The notation used here is fairly standard except where otherwise stated. $\mathbb{R}^{n}$ and $\mathbb{R}^{n \times m}$ denote, respectively, the $n$-dimensional Euclidean space and the set of all $n \times m$ real matrices. $l_{2}[0, \infty)$ is the space of square summable sequences. The notation $X \geq Y$ (respectively, $X>Y$ ), where $X$ and $Y$ are real symmetric matrices, means that $X-Y$ is positive semi-definite (respectively, positive definite). $\operatorname{Prob}\{\cdot\}$ means the occurrence probability of the event "." and $\mathbb{E}\{\cdot\}$ stands for the expectation of the stochastic variable "." with respect to the given probability measure Prob. 0 and $I$ denote, respectively, the zero matrix of compatible dimensions and the identity matrix of compatible dimensions. In symmetric block matrices or complex matrix expressions, we utilize asterisk $*$ to represent a term that is induced by symmetry, and $\operatorname{diag}\{\cdots\}$ stands for a block-diagonal matrix. $\operatorname{col}\{\cdots\}$ represents a column vector composed of elements. $\|\bullet\|$ refers to the Euclidean norm for vectors. $\lfloor\star\rfloor$ is the floor function which is the largest integer not greater than $\star$. Matrices, if not explicitly specified, are assumed to have compatible dimensions. 


\section{INTRODUCTION OF NETWORKED MULTI-RATE SYSTEMS}

Consider the following class of discrete time systems with randomly occurring faults:

$$
\begin{aligned}
x\left(T_{k+1}\right) & =A x\left(T_{k}\right)+B_{1} \omega\left(T_{k}\right)+\alpha\left(T_{k}\right) B_{2} f\left(T_{k}\right) \\
y\left(t_{k}\right) & =C x\left(t_{k}\right), \quad k=0,1,2, \cdots
\end{aligned}
$$

where $x\left(T_{k}\right) \in \mathbb{R}^{n_{x}}$ represents the state vector, $y\left(t_{k}\right) \in \mathbb{R}^{n_{y}}$ is the ideal measurement, $\omega\left(T_{k}\right) \in \mathbb{R}^{n_{\omega}}$ is the disturbance input which belongs to $\ell_{2}[0, \infty)$, and $f\left(T_{k}\right) \in \mathbb{R}^{n_{f}}$ is the fault signal to be detected. $A, B_{1}$, $B_{2}$ and $C$ are constant matrices with appropriate dimensions.

The sampling period of system (1) is denoted by $h \triangleq T_{k+1}-T_{k}$. For simplicity, it is assumed that the measurement period is integer multiples of the system (2), i.e. $t_{k+1}-t_{k} \triangleq b h$, where $b$ is a positive integer. An illustration of the multi-rate sampled-data systems is shown in Fig. 1 where $b=3, T_{k}$ are the updating instants for system states and $t_{k}$ are the updating instants for system measurements.

The stochastic variable $\alpha\left(T_{k}\right)$ is used to govern the random behaviour of the fault occurrence, which is a Bernoulli distributed white-noise sequence taking values on 0 or 1 with the following probabilities:

$$
\operatorname{Prob}\left\{\alpha\left(T_{k}\right)=1\right\}=\bar{\alpha}, \quad \operatorname{Prob}\left\{\alpha\left(T_{k}\right)=0\right\}=1-\bar{\alpha} .
$$

In comparison with the wired NCSs, the wireless NCSs are susceptible to fading effect because of multipath propagation or shadowing from obstacles affecting the wave propagation [12], [13]. In this paper, the actually received measurement signal with probabilistic fading channels is described by

$$
\bar{y}\left(t_{k}\right)=\sum_{s=0}^{\ell\left(t_{k}\right)} \beta_{s}\left(t_{k}\right) y\left(t_{k}-s b h\right)
$$

where $\ell\left(t_{k}\right)=\min \left\{\ell,\left\lfloor\frac{t_{k}}{b h}\right\rfloor\right\}$ with $\ell$ being a given positive scalar denoting the number of paths. $\bar{y}\left(t_{k}\right) \in \mathbb{R}^{n_{y}}$ is the measurement output through fading channels. $\beta_{s}\left(t_{k}\right)\left(s=0,1, \ldots, \ell\left(t_{k}\right)\right)$ are assumed to be mutually independent channel coefficients having probability density functions $q\left(\beta_{s}\right)$ on the interval $[0,1]$ with known mathematical expectations $\bar{\beta}_{s}$ and variances $\tilde{\bar{\beta}}_{s}^{2}$.

Remark 1: In a networked environment, the faults could occur in a random way due to a variety of reasons such as limited bandwidth of the communication channels, random fluctuation of the network load, unreliability of the wireless links with large distances, as well as the fading measurement signals. The network-induced fault can be modelled in (1) whose probability distribution information can be specified a prior through statistical tests. Note that both the time-delays and packet dropouts can be described by this kind of fading model.

It can be seen that (1) evolves with a constant period $h$, while the fading measurement dynamics (3) is generated with a slower period bh. Accordingly, (1) and (3) is essentially a multi-rate sampled-data

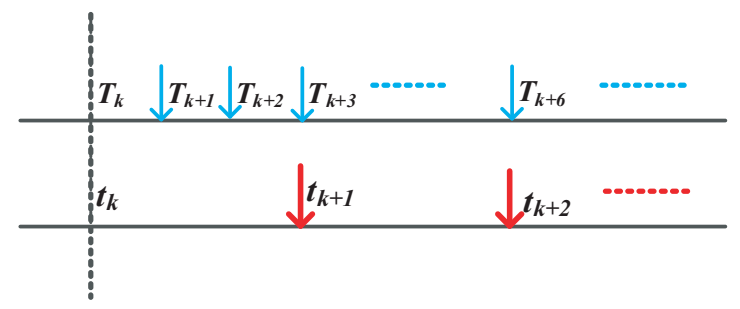

Fig. 1. An illustration of the multi-rate sampled-data system with $b=3$. 
(MRSD) system model. Note that it is mathematically difficult to handle the FD problem directly for such kind of MRSD system. In the next section, we are going to convert the resulting MRSD system into a single-rate system for technical convenience.

\section{MODEL OF THE NETWORKED MULTI-RATE SYSTEMS}

The following assumptions are needed in the derivation of the main results.

Assumption 1: The mutually independent channel coefficients $\beta_{s}\left(t_{k}\right)(s=0,1, \cdots, \ell)$ are independent of the random variable $\alpha\left(T_{k}\right)$ governing the fault occurrence.

Assumption 2: In this paper, for the purpose of simplicity, for $-\ell \leq i \leq-1$, we assume that $x(i)=0$ and $\operatorname{col}\{\omega(i), f(i)\}=0$. Without loss of generality, we also assume that $\ell+1 \leq b$.

By applying the relation (1) recursively, one obtains a new system with time scale $t_{k}$ as follows:

$$
x\left(t_{k+1}\right)=A^{b} x\left(t_{k}\right)+\bar{A}_{11} \bar{\omega}\left(t_{k}\right)+\bar{A}_{12} \bar{f}\left(t_{k}\right)+\sum_{i=0}^{b-1} \tilde{\alpha}\left(t_{k}+i h\right) A^{b-1-i} B_{2} f\left(t_{k}+i h\right)
$$

where

$$
\begin{aligned}
\bar{\omega}\left(t_{k}\right) & \triangleq \operatorname{col}\left\{\omega\left(t_{k}\right), \omega\left(t_{k}+h\right), \cdots, \omega\left(t_{k}+(b-1) h\right)\right\}, \\
\bar{f}\left(t_{k}\right) & \triangleq \operatorname{col}\left\{f\left(t_{k}\right), f\left(t_{k}+h\right), \cdots, f\left(t_{k}+(b-1) h\right)\right\}, \\
\tilde{\alpha}\left(t_{k}+i h\right) & \triangleq \alpha\left(t_{k}+i h\right)-\bar{\alpha}(i=0,1,2, \cdots, b-1), \\
\bar{A}_{11} & \triangleq\left[\begin{array}{lll}
A^{b-1} B_{1} & A^{b-2} B_{1} \cdots A B_{1} & B_{1}
\end{array}\right], \\
\bar{A}_{12} & \triangleq\left[\begin{array}{lll}
\bar{\alpha} A^{b-1} B_{2} & \bar{\alpha} A^{b-2} B_{2} \cdots \bar{\alpha} A B_{2} & \bar{\alpha} B_{2}
\end{array}\right] .
\end{aligned}
$$

Consider the following observer-based fault detection filter

$$
\left\{\begin{aligned}
\hat{x}\left(t_{k+1}\right) & =A^{b} \hat{x}\left(t_{k}\right)+L\left(\bar{y}\left(t_{k}\right)-C \hat{x}\left(t_{k}\right)\right) \\
r\left(t_{k}\right) & =V\left(\bar{y}\left(t_{k}\right)-C \hat{x}\left(t_{k}\right)\right)
\end{aligned}\right.
$$

where $\hat{x}\left(t_{k}\right) \in \mathbb{R}^{n_{\hat{x}}}$ is the estimated state, $r\left(t_{k}\right) \in \mathbb{R}^{n_{r}}$ is the residual that is compatible with the fault vector, and the $L$ and $V$ are the appropriately dimensioned fault detection filter gain matrices to be designed. In our present work, it is intended to make the error between the residual signal $r\left(t_{k}\right)$ and the fault signal $f\left(t_{k}\right)$ as small as possible in $H_{\infty}$ framework.

Letting $e\left(t_{k}\right) \triangleq x\left(t_{k}\right)-\hat{x}\left(t_{k}\right), \bar{x}\left(t_{k}\right) \triangleq \operatorname{col}\left\{x\left(t_{k}\right), x\left(t_{k}-h\right), \cdots, x\left(t_{k}-(b-1) h\right)\right\}$ and $\tilde{\beta}_{s}\left(t_{k}\right) \triangleq$ $\beta_{s}\left(t_{k}\right)-\bar{\beta}_{s}$, the error dynamics for the fault detection filter can be obtained from (4)-(5) and Assumption 2 as follows:

$$
\left\{\begin{aligned}
e\left(t_{k+1}\right)= & \left(A^{b}-L C\right) e\left(t_{k}\right)+\bar{A}_{11} \bar{\omega}\left(t_{k}\right)+\bar{A}_{12} \bar{f}\left(t_{k}\right)+L C x\left(t_{k}\right) \\
& -\sum_{s=0}^{\ell} \tilde{\beta}_{s}\left(t_{k}\right) L C x\left(t_{k}-s b h\right)-\sum_{s=0}^{\ell} \bar{\beta}_{s} L C x\left(t_{k}-s b h\right) \\
& +\sum_{i=0}^{b-1} \tilde{\alpha}\left(t_{k}+i h\right) A^{b-1-i} B_{2} f\left(t_{k}+i h\right) \\
r\left(t_{k}\right)= & V C e\left(t_{k}\right)-V C x\left(t_{k}\right)+\sum_{s=0}^{\ell} \bar{\beta}_{s} V C x\left(t_{k}-s b h\right) \\
& +\sum_{s=0}^{\ell} \tilde{\beta}_{s}\left(t_{k}\right) V C x\left(t_{k}-s b h\right)
\end{aligned}\right.
$$


On the other hand, with similar procedure for obtaining (4), we have

$$
\left\{\begin{array}{c}
x\left(t_{k+1}-h\right)=A^{b-1} x\left(t_{k}\right)+\bar{A}_{21} \bar{\omega}\left(t_{k}\right)+\bar{A}_{22} \bar{f}\left(t_{k}\right) \\
+\sum_{i=0}^{b-2} \tilde{\alpha}\left(t_{k}+i h\right) A^{b-2-i} B_{2} f\left(t_{k}+i h\right) \\
\ldots \\
x\left(t_{k+1}-(b-1) h\right)= \\
\cdots \\
+\bar{A}_{b 2} \bar{f}\left(t_{k}\right)+\bar{A}_{b 1} \bar{\omega}\left(t_{k}\right)
\end{array}\right.
$$

where

$$
\begin{aligned}
\bar{A}_{21} & \triangleq\left[\begin{array}{lll}
A^{b-2} B_{1} & A^{b-3} B_{1} \cdots B_{1} & 0
\end{array}\right], \cdots, \bar{A}_{(b-1) 1} \triangleq\left[\begin{array}{lll}
A B_{1} & B_{1} \cdots 0 & 0
\end{array}\right], \\
\bar{A}_{b 1} & \triangleq\left[\begin{array}{llll}
B_{1} & 0 \cdots 0 & 0
\end{array}\right], \bar{A}_{22} \triangleq\left[\begin{array}{llll}
\bar{\alpha} A^{b-2} B_{2} & \bar{\alpha} A^{b-3} B_{2} \cdots \bar{\alpha} B_{2} & 0
\end{array}\right], \cdots, \\
\bar{A}_{(b-1) 2} & \triangleq\left[\begin{array}{llll}
\bar{\alpha} A B_{2} & \bar{\alpha} B_{2} \cdots 0 & 0
\end{array}\right], \bar{A}_{b 2} \triangleq\left[\begin{array}{llll}
\bar{\alpha} B_{2} & 0 \cdots 0 & 0
\end{array}\right] .
\end{aligned}
$$

For convenience of later analysis, we denote

$$
\begin{aligned}
& \eta\left(t_{k}\right) \triangleq \operatorname{col}\left\{e\left(t_{k}\right), \bar{x}\left(t_{k}\right), \bar{x}\left(t_{k}-b h\right), \cdots, \bar{x}\left(t_{k}-\ell b h\right)\right\}, r_{e}\left(t_{k}\right) \triangleq r\left(t_{k}\right)-f\left(t_{k}\right), \\
& \mathcal{I} \triangleq \operatorname{col}\{I, \underbrace{0, \cdots, 0}_{(\ell+1) b}\}, \bar{A} \triangleq \operatorname{col}\{\left(1-\bar{\beta}_{0}\right) L C, A^{b}, A^{b-1}, \cdots, A, \underbrace{0, \cdots, 0}_{\ell b}\}, \\
& \bar{B}_{1} \triangleq \operatorname{col}\{A^{b-1} B_{2}, \underbrace{A^{b-1} B_{2}, A^{b-2} B_{2}, \cdots, A B_{2}, B_{2}}_{b}, \underbrace{0, \cdots, 0}_{\ell b}\}, \\
& \bar{B}_{2} \triangleq \operatorname{col}\{A^{b-2} B_{2}, \underbrace{A^{b-2} B_{2}, A^{b-3} B_{2}, \cdots, B_{2}, 0}_{b}, \underbrace{0, \cdots, 0}_{\ell b}\}, \cdots \\
& \bar{B}_{b} \triangleq \operatorname{col}\{B_{2}, \underbrace{B_{2}, 0, \cdots, 0,0}_{b}, \underbrace{0, \cdots, 0}_{\ell b}\}
\end{aligned}
$$

Then, by using the lifting technique, the augmented system resulting from (4), (6) and (7) can be written as

$$
\left\{\begin{aligned}
\eta\left(t_{k+1}\right)=(\mathcal{A} & \left.+\sum_{s=0}^{\ell} \tilde{\beta}_{s}\left(t_{k}\right) \tilde{\mathcal{A}}_{s}\right) \eta\left(t_{k}\right)+\mathcal{B}_{1} \bar{\omega}\left(t_{k}\right) \\
& +\left(\mathcal{D}+\sum_{i=0}^{b-1} \tilde{\alpha}\left(t_{k}+i h\right) \tilde{\mathcal{D}}_{i}\right) \bar{f}\left(t_{k}\right) \\
r_{e}\left(t_{k}\right)= & \left(\mathcal{C}+\sum_{s=0}^{\ell} \tilde{\beta}_{s}\left(t_{k}\right) \tilde{\mathcal{C}}_{s}\right) \eta\left(t_{k}\right)+\mathcal{B}_{2} \bar{f}\left(t_{k}\right)
\end{aligned}\right.
$$

where

$$
\begin{aligned}
& \mathcal{A} \triangleq[\left(A^{b}-L C\right) \mathcal{I} \bar{A}-\bar{\beta}_{1} L C \mathcal{I}-\bar{\beta}_{2} L C \mathcal{I} \cdots-\bar{\beta}_{\ell} L C \mathcal{I} \underbrace{0 \cdots 0}_{\ell b}], \\
& \tilde{\mathcal{A}}_{s} \triangleq[\underbrace{0 \cdots 0}_{s+1} \operatorname{\cdots i} \underbrace{0 \cdots 0}_{(\ell+1) b-s-1}], \\
& \mathcal{B}_{1} \triangleq \operatorname{col}\{\bar{A}_{11}, \bar{A}_{11}, \bar{A}_{21}, \cdots, \bar{A}_{(b-1) 1}, \bar{A}_{b 1}, \underbrace{0, \cdots, 0}_{\ell b}\},
\end{aligned}
$$




$$
\begin{aligned}
\mathcal{D} \triangleq & \operatorname{col}\{\bar{A}_{12}, \bar{A}_{12}, \bar{A}_{22}, \cdots, \bar{A}_{(b-1) 2}, \bar{A}_{b 2}, \underbrace{0, \cdots, 0}_{\ell b}\}, \\
\tilde{\mathcal{D}}_{i} \triangleq & {[\underbrace{0 \cdots 0}_{i} \bar{B}_{i+1} \underbrace{0 \cdots 0}_{b-i-1}], } \\
\mathcal{C} \triangleq & {[V C-\left(1-\bar{\beta}_{0}\right) V C \bar{\beta}_{1} V C \bar{\beta}_{2} V C \cdots \bar{\beta}_{\ell} V C \underbrace{0 \cdots 0}_{\ell b}], } \\
\tilde{\mathcal{C}}_{s} \triangleq & {[\underbrace{0 \cdots 0}_{s+1} V C \underbrace{0 \cdots 0}_{(\ell+1) b-s-1}], \mathcal{B}_{2} \triangleq[-I \underbrace{0 \cdots 0}_{b-1}], } \\
& (s=0,1, \cdots, \ell ; i=0,1, \cdots b-1) .
\end{aligned}
$$

Remark 2: By using the lifting technique, the model (8) for NMSs is obtained. Comparing with the fault detection models of the MRSD system in [18]-[20], the model (8) exhibits two distinguished features: i) both the fading measurements and randomly occurring faults are considered and therefore the model (8) is quite comprehensive to better reflect the networked environment; ii) the introduction of the stochastic coefficients in model (3) results in significant delays in the overall dynamics governed by (8). Note that the communication delay issues have not been considered in [18]-[20].

Before proceeding further, we introduce the following definition.

Definition 1: The augmented system (8) is said to be exponentially mean-square stable if, with $\bar{\omega}\left(t_{k}\right)=$ 0 and $\bar{f}\left(t_{k}\right)=0$, there exist scalars $\delta>0$ and $\varrho \in(0,1)$ such that

$$
\mathbb{E}\left\{\left\|\eta\left(t_{k}\right)\right\|^{2}\right\} \leq \delta \varrho^{t_{k}} \mathbb{E}\left\{\left\|\eta\left(t_{0}\right)\right\|^{2}\right\}, \quad \forall \eta\left(t_{0}\right) \in \mathbb{R}^{(b+1) n_{x}}
$$

The purpose of this paper is to design the observer-based fault detection filters such that the following requirements are met simultaneously:

(a) the augmented system (8) is exponentially mean-square stable;

(b) under the zero-initial condition, the error $r_{e}\left(t_{k}\right)$ between the residual and the fault estimate satisfies

$$
\sum_{k=0}^{\infty} \mathbb{E}\left\{\left\|r_{e}\left(t_{k}\right)\right\|^{2}\right\}<\gamma^{2} \sum_{k=0}^{\infty}\left(\left\|\bar{\omega}\left(t_{k}\right)\right\|^{2}+\left\|\bar{f}\left(t_{k}\right)\right\|^{2}\right)
$$

for any nonzero $\bar{\omega}\left(t_{k}\right)$ or $\bar{f}\left(t_{k}\right)$, where scalar $\gamma>0$ is a given disturbance attenuation level.

For the fault detection purpose, we adopt the threshold $J_{t h}$ and the residual evaluation function $J\left(t_{k}\right)$ as follows:

$$
J\left(t_{k}\right)=\left\{\sum_{h=t_{k_{0}}}^{t_{k}} r^{T}(h) r(h)\right\}^{\frac{1}{2}}, \quad J_{t h}=\sup _{\substack{\bar{\omega}\left(t_{k}\right) \in \ell_{2} \\ \bar{f}\left(t_{k}\right)=0}} \mathbb{E}\left\{J\left(t_{k}\right)\right\}
$$

where $t_{k_{0}}$ denotes the initial evaluation time instant and $t_{k}-t_{k_{0}}$ denotes the evaluation time steps.

The occurrence of faults can be detected by comparing $J\left(t_{k}\right)$ with $J_{t h}$ according to the following test rule:

$$
\left\{\begin{array}{l}
J\left(t_{k}\right) \geq J_{t h} \Longrightarrow \text { alarm for fault } \\
J\left(t_{k}\right)<J_{t h} \Longrightarrow \text { no fault }
\end{array}\right.
$$

Remark 3: As is discussed in [23], depending on the type of the system under consideration, there exist two residual evaluation strategies, i.e. the statistic testing and norm-based residual evaluation. For the norm-based residual evaluation, the well-established robust control theory can be used to compute 
the threshold, therefore, it is widely adopted. On the other hand, from the engineering viewpoint, the determination of a threshold is to find out the tolerant limit for disturbances and model uncertainties under fault-free operation conditions. There are some factors such as the dynamics of the residual generator as well as the bounds of the unknown inputs and model uncertainties, they all significantly influence this procedure. As a result, false alarm and missed detection are two common phenomenon in fault diagnosis.

\section{MAin RESUlTS}

In this section, by resorting to the stochastic analysis techniques, we shall provide the $H_{\infty}$ performance analysis result for the augmented system (8) and then proceed with the subsequent fault detection filter design stage.

Theorem 1: Let the disturbance attenuation level $\gamma>0$ and the fault detection filter parameters $L$ and $V$ be given. The augmented system (8) is exponentially mean-square stable while achieving the $H_{\infty}$ performance constraint (9) if there exists matrix $P$ such that the following matrix inequality holds:

$$
\hat{\Phi} \triangleq\left[\begin{array}{ccc}
\bar{\Phi}_{11} & \bar{\Phi}_{12} & \bar{\Phi}_{13} \\
* & -I & 0 \\
* & * & -I
\end{array}\right]<0
$$

where

$$
\begin{aligned}
& \bar{\Phi}_{11} \triangleq\left[\begin{array}{ccc}
\Gamma & \mathcal{A}^{T} P \mathcal{B}_{1} & \mathcal{A}^{T} P \mathcal{D}+\mathcal{C}^{T} \mathcal{B}_{2} \\
* & \mathcal{B}_{1}^{T} P \mathcal{B}_{1}-\gamma^{2} I & \mathcal{B}_{1}^{T} P \mathcal{D} \\
* & * & \Phi_{33}
\end{array}\right], \\
& \Gamma \triangleq \sum_{s=0}^{\ell} \tilde{\bar{\beta}}_{s}^{2} \tilde{\mathcal{A}}_{s}^{T} P \tilde{\mathcal{A}}_{s}+\mathcal{A}^{T} P \mathcal{A}-P, \bar{\Phi}_{12} \triangleq \operatorname{col}\left\{\mathcal{C}^{T}, 0,0\right\} \\
& \bar{\Phi}_{13} \triangleq \operatorname{col}\left\{\hat{\tilde{\mathcal{C}}}^{T}, 0,0\right\}, \hat{\tilde{\mathcal{C}}}^{T} \triangleq\left[\tilde{\bar{\beta}}_{0} \tilde{\mathcal{C}}_{0}^{T}\right.\left.\tilde{\bar{\beta}}_{1} \tilde{\mathcal{C}}_{1}^{T} \ldots \tilde{\bar{\beta}}_{\ell} \tilde{\mathcal{C}}_{\ell}^{T}\right], \\
& \Phi_{33} \triangleq \sum_{i=0}^{b-1} \check{\alpha}^{2} \tilde{\mathcal{D}}_{i}^{T} P \tilde{\mathcal{D}}_{i}+\mathcal{D}^{T} P \mathcal{D}+\mathcal{B}_{2}^{T} \mathcal{B}_{2}-\gamma^{2} I .
\end{aligned}
$$

Proof: Choose the following Lyapunov function:

$$
V\left(\eta\left(t_{k}\right)\right)=\eta^{T}\left(t_{k}\right) P \eta\left(t_{k}\right)
$$

By calculating the difference of $V\left(\eta\left(t_{k}\right)\right)$ along the trajectory of the augmented system (8) with $\bar{\omega}\left(t_{k}\right)=$ 0 and $\bar{f}\left(t_{k}\right)=0$, and taking the mathematical expectation, one has

$$
\begin{aligned}
\mathbb{E}\left(\Delta V\left(\eta\left(t_{k}\right)\right)\right) & =\mathbb{E}\left\{\eta^{T}\left(t_{k+1}\right) P \eta\left(t_{k+1}\right)-\eta^{T}\left(t_{k}\right) P \eta\left(t_{k}\right)\right\} \\
& =\mathbb{E}\left\{\eta^{T}\left(t_{k}\right)\left(\left(\mathcal{A}+\sum_{s=0}^{\ell} \tilde{\beta}_{s}\left(t_{k}\right) \tilde{\mathcal{A}}_{s}\right)^{T} P\left(\mathcal{A}+\sum_{s=0}^{\ell} \tilde{\beta}_{s}\left(t_{k}\right) \tilde{\mathcal{A}}_{s}\right)-P\right) \eta\left(t_{k}\right)\right\} \\
& =\eta^{T}\left(t_{k}\right)\left(\mathcal{A}^{T} P \mathcal{A}-P+\sum_{s=0}^{\ell} \tilde{\beta}_{s}^{2} \tilde{\mathcal{A}}_{s}^{T} P \tilde{\mathcal{A}}_{s}\right) \eta\left(t_{k}\right) \\
& =\eta^{T}\left(t_{k}\right) \Gamma \eta\left(t_{k}\right)
\end{aligned}
$$

It follows from (11) that $\Gamma<0$ and, subsequently,

$$
\mathbb{E}\left(\Delta V\left(\eta\left(t_{k}\right)\right)\right) \leq-\lambda_{\min }(-\Gamma)\left\|\eta\left(t_{k}\right)\right\|^{2}
$$


By following the similar analysis in [5], the augmented system (8) is exponentially mean-square stable.

Finally, let us consider the $H_{\infty}$ performance of the overall estimation dynamics. For this purpose, we introduce the following index:

$$
J_{n} \triangleq \mathbb{E}\left\{\sum_{k=0}^{n}\left\|r_{e}\left(t_{k}\right)\right\|^{2}-\sum_{k=0}^{n} \gamma^{2}\left(\left\|\bar{\omega}\left(t_{k}\right)\right\|^{2}+\left\|\bar{f}\left(t_{k}\right)\right\|^{2}\right)\right\}
$$

Under the zero-initial condition, it follows from (14) that

$$
\begin{aligned}
J_{n} \triangleq & \mathbb{E}\left\{\sum_{k=0}^{n}\left\|r_{e}\left(t_{k}\right)\right\|^{2}-\sum_{k=0}^{n} \gamma^{2}\left(\left\|\bar{\omega}\left(t_{k}\right)\right\|^{2}+\left\|\bar{f}\left(t_{k}\right)\right\|^{2}\right)\right\} \\
\leq & \sum_{k=0}^{n} \mathbb{E}\left\{\left\|r_{e}\left(t_{k}\right)\right\|^{2}-\gamma^{2}\left(\left\|\bar{\omega}\left(t_{k}\right)\right\|^{2}+\left\|\bar{f}\left(t_{k}\right)\right\|^{2}\right)+\Delta V\left(\eta\left(t_{k}\right)\right)\right\}-\mathbb{E}\left\{V\left(\eta\left(t_{n+1}\right)\right\}\right. \\
\leq & \sum_{k=0}^{n} \mathbb{E}\left\{\left\|r_{e}\left(t_{k}\right)\right\|^{2}-\gamma^{2}\left(\left\|\bar{\omega}\left(t_{k}\right)\right\|^{2}+\left\|\bar{f}\left(t_{k}\right)\right\|^{2}\right)+\Delta V\left(\eta\left(t_{k}\right)\right)\right\} \\
= & \sum_{k=0}^{n}\left\{\eta ^ { T } ( t _ { k } ) \left[\sum_{s=0}^{\ell} \tilde{\bar{\beta}}_{s}^{2} \tilde{\mathcal{A}}_{s}^{T} P \tilde{\mathcal{A}}_{s}+\mathcal{A}^{T} P \mathcal{A}+\sum_{s=0}^{\ell} \tilde{\bar{\beta}}_{s}^{2} \tilde{\mathcal{C}}_{s}^{T} \tilde{\mathcal{C}}_{s}\right.\right. \\
& \left.+\mathcal{C}^{T} \mathcal{C}-P\right] \eta\left(t_{k}\right)+2 \eta^{T}\left(t_{k}\right)\left[\mathcal{A}^{T} P \mathcal{D}+\mathcal{C}^{T} \mathcal{B}_{2}\right] \bar{f}\left(t_{k}\right) \\
& +2 \eta^{T}\left(t_{k}\right) \mathcal{A}^{T} P \mathcal{B}_{1} \bar{\omega}\left(t_{k}\right)+2 \bar{\omega}^{T}\left(t_{k}\right) \mathcal{B}_{1}^{T} P \mathcal{D} \bar{f}\left(t_{k}\right) \\
& +\bar{f}^{T}\left(t_{k}\right)\left[\sum_{i=0}^{b-1} \check{\alpha}^{2} \tilde{\mathcal{D}}_{i}^{T} P \tilde{\mathcal{D}}_{i}+\mathcal{D}^{T} P \mathcal{D}+\mathcal{B}_{2}^{T} \mathcal{B}_{2}-\gamma^{2} I\right] \bar{f}\left(t_{k}\right) \\
& \left.+\bar{\omega}^{T}\left(t_{k}\right)\left[\mathcal{B}_{1}^{T} P \mathcal{B}_{1}-\gamma^{2} I\right] \bar{\omega}\left(t_{k}\right)\right\} \\
= & \sum_{k=0}^{n}\left\{\vartheta^{T}\left(t_{k}\right) \Phi \vartheta\left(t_{k}\right)\right\} \\
= & \sum_{k=0}^{n}\left\{\vartheta^{T}\left(t_{k}\right)\left(\bar{\Phi}_{11}+\tilde{\Phi}\right) \vartheta\left(t_{k}\right)\right\}
\end{aligned}
$$

where

$$
\begin{gathered}
\vartheta\left(t_{k}\right) \triangleq \operatorname{col}\left\{\eta\left(t_{k}\right), \bar{\omega}\left(t_{k}\right), \bar{f}\left(t_{k}\right)\right\}, \mathbb{E}\left\{\tilde{\alpha}^{2}\left(t_{k}+i h\right)\right\}=(\sqrt{\bar{\alpha}(1-\bar{\alpha})})^{2} \triangleq \check{\alpha}^{2}, \\
\Phi \triangleq \bar{\Phi}_{11}+\tilde{\Phi}, \tilde{\Phi} \triangleq \operatorname{diag}\left\{\sum_{s=0}^{\ell} \tilde{\bar{\beta}}_{s}^{2} \tilde{\mathcal{C}}_{s}^{T} \tilde{\mathcal{C}}_{s}+\mathcal{C}^{T} \mathcal{C}, 0,0\right\}=\bar{\Phi}_{12} \bar{\Phi}_{12}^{T}+\bar{\Phi}_{13} \bar{\Phi}_{13}^{T}
\end{gathered}
$$

By using the Schur Complement Lemma to (11), we have

$$
\hat{\Phi}=\bar{\Phi}_{11}+\bar{\Phi}_{12} \bar{\Phi}_{12}^{T}+\bar{\Phi}_{13} \bar{\Phi}_{13}^{T}<0
$$

that is $\bar{\Phi}_{11}+\tilde{\Phi}<0$, therefore, we obtain the following relation from (15)

$$
\mathbb{E}\left(\Delta V\left(\eta\left(t_{k}\right)\right)\right)+\mathbb{E}\left(\left\|r_{e}\left(t_{k}\right)\right\|^{2}\right)-\gamma^{2}\left(\left\|\bar{\omega}\left(t_{k}\right)\right\|^{2}+\left\|\bar{f}\left(t_{k}\right)\right\|^{2}\right)<0
$$

for all nonzero $\bar{\omega}\left(t_{k}\right)$ and $\bar{f}\left(t_{k}\right)$. Considering zero initial condition, the inequality (17) implies that

$$
\sum_{k=0}^{n} \mathbb{E}\left\{\left\|r_{e}\left(t_{k}\right)\right\|^{2}\right\}<\gamma^{2} \sum_{k=0}^{n}\left(\left\|\bar{\omega}\left(t_{k}\right)\right\|^{2}+\left\|\bar{f}\left(t_{k}\right)\right\|^{2}\right)
$$


Letting $n \rightarrow \infty$, it follows from the aforementioned inequality that

$$
\sum_{k=0}^{\infty} \mathbb{E}\left\{\left\|r_{e}\left(t_{k}\right)\right\|^{2}\right\}<\gamma^{2} \sum_{k=0}^{\infty}\left(\left\|\bar{\omega}\left(t_{k}\right)\right\|^{2}+\left\|\bar{f}\left(t_{k}\right)\right\|^{2}\right)
$$

which is (9). The proof is now complete.

Having established the analysis results, we are now ready to deal with the filter design problem. In the following theorem, a sufficient condition is provided for the existence of the desired $H_{\infty}$ multi-rate fault detection filter. For technical convenience, we denote

$$
\begin{aligned}
& \overline{\mathcal{A}}_{10}^{T} \triangleq \operatorname{col}\left\{-\bar{\beta}_{1} C^{T} \bar{L}^{T},-\bar{\beta}_{2} C^{T} \bar{L}^{T}, \cdots,-\bar{\beta}_{\ell} C^{T} \bar{L}^{T}\right\}, \\
& \overline{\mathcal{A}}_{1}^{T} \triangleq \operatorname{col}\{\left(A^{b}\right)^{T} P_{1}-C^{T} \bar{L}^{T},\left(1-\bar{\beta}_{0}\right) C^{T} \bar{L}^{T}, \overline{\mathcal{A}}_{10}^{T}, \underbrace{0, \cdots, 0}_{\ell b}\}, \\
& \overline{\mathcal{A}}_{i}^{T} \triangleq \operatorname{col}\{\left(A^{b+2-i}\right)^{T} P_{i}, \underbrace{0, \cdots, 0}_{(\ell+1) b}\}, \hat{\overline{\mathcal{A}}}^{T} \triangleq[\overline{\mathcal{A}}_{1}^{T} \overline{\mathcal{A}}_{2}^{T} \cdots \overline{\mathcal{A}}_{b+1}^{T} \underbrace{0 \cdots 0}_{\ell b}], \\
& \mathcal{X}_{j}^{T} \triangleq \operatorname{col}\{\underbrace{0, \cdots, 0}_{j+1},-C^{T} \bar{L}^{T}, \underbrace{0, \cdots, 0}_{b-1-j}\}, \overline{\tilde{\mathcal{A}}}_{j}^{T} \triangleq[\mathcal{X}_{j}^{T} \underbrace{0 \cdots 0}_{b}],
\end{aligned}
$$

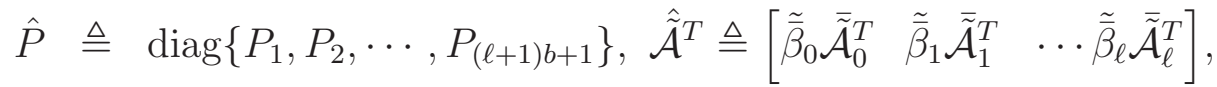

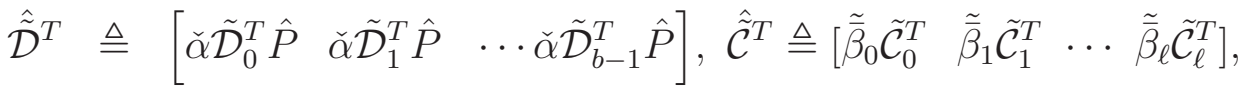

$$
\begin{aligned}
& (i=2,3, \cdots, b+1 ; j=0,1,2, \cdots, \ell) \text {. }
\end{aligned}
$$

Theorem 2: For the given disturbance attenuation level $\gamma>0$, the augmented system (8) is exponentially mean-square stable while achieving the performance constraint (9) for any nonzero $\bar{\omega}\left(t_{k}\right)$ and $\bar{f}\left(t_{k}\right)$ if there exist matrices $\bar{L}, \bar{V}$ and $P_{i}>0(i=1,2, \cdots,(\ell+1) b+1)$ such that the following linear matrix inequality (LMI) holds:

$$
\Xi \triangleq\left[\begin{array}{ccc}
\bar{\Xi}_{11} & \Xi_{12} & \bar{\Xi}_{13} \\
* & \Xi_{22} & 0 \\
* & * & \bar{\Xi}_{33}
\end{array}\right]<0
$$

where

$$
\begin{aligned}
& \Xi_{11} \triangleq \operatorname{diag}\left\{-\hat{P},-\gamma^{2} I,-\gamma^{2} I\right\}, \Xi_{12} \triangleq\left[\begin{array}{cc}
\hat{\tilde{\mathcal{C}}}^{T} & \mathcal{C}^{T} \\
0 & 0 \\
0 & \mathcal{B}_{2}^{T}
\end{array}\right], \\
& \bar{\Xi}_{13} \triangleq\left[\begin{array}{ccc}
\tilde{\tilde{\mathcal{A}}}^{T} & 0 & \hat{\overline{\mathcal{A}}}^{T} \\
0 & 0 & \mathcal{B}_{1}^{T} \hat{P} \\
0 & \hat{\tilde{\mathcal{D}}}^{T} & \mathcal{D}^{T} \hat{P}
\end{array}\right], \Xi_{22} \triangleq \operatorname{diag}\{-I, \cdots,-I\}, \\
& \bar{\Xi}_{33} \triangleq \operatorname{diag}\{-\hat{P}, \cdots,-\hat{P}\},
\end{aligned}
$$

and other corresponding matrices are defined in Theorem 1. Furthermore, if the inequality (18) is feasible, the desired fault detection filter gain can be determined by

$$
L=P_{1}^{-1} \bar{L}, V=\bar{V} .
$$


Proof: By using the Schur Complement Lemma, (11) is equivalent to the following inequality:

$$
\Xi=\left[\begin{array}{ccc}
\Xi_{11} & \Xi_{12} & \Xi_{13} \\
* & \Xi_{22} & 0 \\
* & * & \Xi_{33}
\end{array}\right]<0
$$

where

$$
\begin{aligned}
& \Xi_{11} \triangleq \operatorname{diag}\left\{-P,-\gamma^{2} I,-\gamma^{2} I\right\}, \quad \Xi_{13} \triangleq\left[\begin{array}{ccc}
\check{\tilde{\mathcal{A}}}^{T} & 0 & \mathcal{A}^{T} P \\
0 & 0 & \mathcal{B}_{1}^{T} P \\
0 & \tilde{\tilde{\mathcal{D}}}^{T} & \mathcal{D}^{T} P
\end{array}\right] \\
& \Xi_{33} \triangleq \operatorname{diag}\{\underbrace{-P, \cdots,-P}_{b+\ell+2}\}, \quad \check{\tilde{\mathcal{A}}}^{T} \triangleq\left[\begin{array}{cccc}
\tilde{\bar{\beta}}_{0} \tilde{\mathcal{A}}_{0}^{T} P & \tilde{\bar{\beta}}_{1} \tilde{\mathcal{A}}_{1}^{T} P & \ldots & \tilde{\bar{\beta}}_{\ell} \tilde{\mathcal{A}}_{\ell}^{T} P
\end{array}\right], \\
& \check{\tilde{\mathcal{D}}}^{T} \triangleq\left[\begin{array}{clll}
\check{\alpha} \tilde{\mathcal{D}}_{0}^{T} P & \check{\alpha} \tilde{\mathcal{D}}_{1}^{T} P & \cdots & \check{\alpha}^{2} \tilde{\mathcal{D}}_{b-1}^{T} P
\end{array}\right] .
\end{aligned}
$$

In order to utilize the Matlab LMI Toolbox to design the fault detection filter effectively, we assume $P$ as $\hat{P}=\operatorname{diag}\left\{P_{1}, P_{2}, \cdots, P_{(\ell+1) b+1}\right\}$, let $\bar{L}=P_{1} L$ and $\bar{V}=V$, then (18) can be obtained and the fault detection filter can be expressed as (19). The proof of this theorem is now complete.

To sum up, the FD problem of networked multi-rate systems can be solved by the following steps:

1) Design the fault detection filter by using Theorem 2.

2) Employ the designed fault detection filters in 1) to produce the residual evaluation function $J\left(t_{k}\right)$ and appropriate threshold $J_{t h}$.

3) Compare the residual evaluation function $J\left(t_{k}\right)$ with the threshold $J_{t h}$ to determine whether there is a fault by using the test rule (10).

4) Determine the fault occurrence time according to $J_{t h}<J\left(t_{k}\right)$ for the first time.

As the special case of NMSs, we now deal with the fault detection filter design problem of networked single-rate systems (NSSs) with network-induced fading measurements and randomly occurring faults. With lifting technique, for system (1)-(3) with $b=1$, choosing observer-based fault detection filter as residual generator (5), and letting

$$
\begin{aligned}
\bar{\omega}\left(T_{k}\right) & \triangleq \operatorname{col}\left\{\omega\left(T_{k}\right), \omega\left(T_{k}-h\right), \cdots, \omega\left(T_{k}-\ell h\right)\right\}, \\
\bar{f}\left(T_{k}\right) & \triangleq \operatorname{col}\left\{f\left(T_{k}\right), f\left(T_{k}-h\right), \cdots, f\left(T_{k}-\ell h\right)\right\}, \\
\bar{e}\left(T_{k}\right) & \triangleq \operatorname{col}\left\{e\left(T_{k}\right), x\left(T_{k}\right), x\left(T_{k}-h\right), \cdots, x\left(T_{k}-\ell h\right)\right\}, \\
e\left(T_{k}\right) & \triangleq x\left(T_{k}\right)-\hat{x}\left(T_{k}\right), \mathcal{J} \triangleq \operatorname{col}\{I, \underbrace{0, \cdots, 0}_{\ell+1}\}, \\
\underline{A}_{i} & \triangleq \operatorname{col}\{\left(1-\bar{\beta}_{i-2}\right) L C, \underbrace{0, \cdots, 0}_{i-2}, A, \underbrace{0, \cdots, 0}_{\ell-i-2}\}, \\
\underline{A}_{1} & \triangleq \operatorname{col}\{A-L C, \underbrace{0, \cdots, 0}_{\ell+1}\}, \mathcal{J} \mathcal{J}_{1} \triangleq \operatorname{col}\{I, I, \underbrace{0, \cdots, 0}_{\ell-1}\}, \\
\mathcal{J}_{j} & \triangleq \operatorname{col}\{\underbrace{0, \cdots, 0}_{j}, I, \underbrace{0, \cdots, 0}_{\ell+1-j}\},(i=2,3, \cdots, \ell+2 ; j=2,3, \cdots, \ell+1),
\end{aligned}
$$


we have the following augmented system:

$$
\left\{\begin{aligned}
\bar{e}\left(T_{k+1}\right)= & \left(\mathscr{A}+\sum_{s=0}^{\ell} \tilde{\beta}_{s}\left(T_{k}\right) \tilde{\mathscr{A}}_{s}\right) \bar{e}\left(T_{k}\right)+\mathscr{B}_{1} \bar{\omega}\left(T_{k}\right) \\
& +\left(\mathscr{D}+\sum_{i=0}^{\ell} \tilde{\alpha}\left(T_{k}-i h\right) \tilde{\mathscr{D}}_{i}\right) \bar{f}\left(T_{k}\right) \\
r_{e}\left(T_{k}\right)= & \left(\mathscr{C}+\sum_{s=0}^{\ell} \tilde{\beta}_{s}\left(T_{k}\right) \tilde{\mathscr{C}}_{s}\right) \bar{e}\left(T_{k}\right)+\mathscr{B}_{2} \bar{f}\left(T_{k}\right)
\end{aligned}\right.
$$

where

$$
\begin{aligned}
& \mathscr{A} \triangleq\left[\begin{array}{llll}
\underline{A}_{1} & \underline{A}_{2} & \cdots & \underline{A}_{\ell+2}
\end{array}\right], \quad \tilde{\mathscr{A}}_{s} \triangleq[\underbrace{0 \cdots 0}_{s+1}-L C \mathcal{J} \underbrace{0 \cdots 0}_{\ell-s}], \\
& \mathscr{B}_{1} \triangleq\left[\begin{array}{llll}
\mathcal{J}_{1} B_{1} & \mathcal{J}_{2} B_{1} & \cdots & \mathcal{J}_{\ell+1} B_{1}
\end{array}\right], \mathscr{D} \triangleq\left[\begin{array}{llll}
\bar{\alpha} \mathcal{J}_{1} B_{2} & \bar{\alpha} \mathcal{J}_{2} B_{2} & \cdots & \bar{\alpha} \mathcal{J}_{\ell+1} B_{2}
\end{array}\right], \\
& \tilde{\mathscr{D}}_{i} \triangleq[\underbrace{0 \cdots 0}_{i} \mathcal{J}_{i+1} B_{2} \underbrace{0 \cdots 0}_{\ell-i}], \tilde{\mathscr{C}}_{s} \triangleq[\underbrace{0 \cdots 0}_{s+1} \quad V C \underbrace{0 \cdots 0}_{\ell-s}], \\
& \mathscr{C} \triangleq\left[\begin{array}{lllll}
V C & -\left(1-\bar{\beta}_{0}\right) V C \mid \bar{\beta}_{1} V C & \ldots & \bar{\beta}_{\ell} V C
\end{array}\right], \\
& \mathscr{B}_{2} \triangleq \operatorname{col}\{-I, \underbrace{0, \cdots, 0}_{\ell}\},(s, i=0,1, \cdots, \ell) .
\end{aligned}
$$

Based on the augmented system (21), by following similar main line of obtaining Theorems 1-2, the fault detection filter of NNSs can be designed by the following Corollary. To facilitate the presentation of Corollary 1, we denote

$$
\begin{aligned}
& \mathcal{Y}_{i} \triangleq \operatorname{col}\{\underbrace{0, \cdots, 0}_{i+1},-C^{T} \vec{L}^{T}, \underbrace{0, \cdots, 0}_{\ell-i}\}, \mathcal{Z}_{i} \triangleq\left[\begin{array}{llll}
\mathcal{Y}_{i} & 0 & \cdots & 0
\end{array}\right], \\
& \overline{\mathscr{A}}_{1} \triangleq \operatorname{col}\left\{A^{T} Q_{1}-C^{T} \vec{L}^{T},\left(1-\bar{\beta}_{0}\right) C^{T} \vec{L}^{T}, \mid-\bar{\beta}_{1} C^{T} \vec{L}^{T}, \cdots, \quad-\bar{\beta}_{\ell} C^{T} \vec{L}^{T}\right\} \text {, } \\
& \overline{\mathscr{A}}_{j} \triangleq \operatorname{col}\{\underbrace{0, \cdots, 0}_{j-1}, A^{T} Q_{2}, \underbrace{0, \cdots, 0}_{\ell+2-j}\}, \quad \hat{\mathscr{A}} \triangleq\left[\begin{array}{llll}
\overline{\mathscr{A}}_{1} & \overline{\mathscr{A}}_{2} & \cdots & \overline{\mathscr{A}}_{\ell+2}
\end{array}\right], \\
& \hat{\tilde{\mathscr{A}}} \triangleq\left[\begin{array}{llll}
\tilde{\bar{\beta}}_{0} \mathcal{Z}_{0} & \tilde{\bar{\beta}}_{1} \mathcal{Z}_{1} & \cdots & \tilde{\bar{\beta}}_{\ell} \mathcal{Z}_{\ell}
\end{array}\right], \hat{Q} \triangleq \operatorname{diag}\left\{Q_{1}, Q_{2}, \cdots, Q_{\ell+2}\right\}, \\
& \hat{\tilde{D}} \triangleq\left[\begin{array}{lllll}
\check{\alpha} \tilde{\mathscr{D}}_{0}^{T} \hat{Q} & \check{\alpha} \tilde{\mathscr{D}}_{1}^{T} \hat{Q} & \cdots & \check{\alpha} \tilde{\mathscr{D}}_{\ell}^{T} \hat{Q}
\end{array}\right], \hat{\mathscr{\mathscr { C }}} \triangleq\left[\begin{array}{llll}
\tilde{\bar{\beta}}_{0} \tilde{\mathscr{C}}_{0}^{T} & \tilde{\bar{\beta}}_{1} \tilde{\mathscr{C}}_{1}^{T} & \cdots & \tilde{\bar{\beta}}_{\ell} \tilde{\mathscr{C}}_{\ell}^{T}
\end{array}\right], \\
& (i=0,1, \cdots, \ell ; j=2,3, \cdots, \ell+2) \text {. }
\end{aligned}
$$

Corollary 1: For the given disturbance attenuation level $\gamma>0$, the augmented system (21) is exponentially mean-square stable while achieving the performance constraint (9) for any nonzero $\bar{\omega}\left(T_{k}\right)$ and $\bar{f}\left(T_{k}\right)$ if there exist matrices $\vec{L}, \vec{V}$ and $Q_{i}>0(i=1,2, \cdots, \ell+2)$ such that the following LMI holds:

$$
\Psi=\left[\begin{array}{ccc}
\Psi_{11} & \Psi_{12} & \Psi_{13} \\
* & \Psi_{22} & 0 \\
* & * & \Psi_{33}
\end{array}\right]<0
$$


where

$$
\begin{aligned}
& \Psi_{11}=\operatorname{diag}\left\{-\hat{Q},-\gamma^{2} I,-\gamma^{2} I\right\}, \quad \Psi_{12}=\left[\begin{array}{cc}
\mathscr{C}^{T} & \hat{\tilde{C}} \\
\mathscr{B}_{2}^{T} & 0 \\
0 & 0
\end{array}\right], \quad \Psi_{13}=\left[\begin{array}{ccc}
\hat{\tilde{A}} & 0 & \hat{\tilde{A}} \\
0 & 0 & \mathscr{D}^{T} \hat{Q} \\
0 & \hat{\tilde{D}} & \mathscr{B}_{1}^{T} \hat{Q}
\end{array}\right], \\
& \Psi_{22}=\operatorname{diag}\{-I, \cdots,-I\}, \Psi_{33}=\operatorname{diag}\{-\hat{Q}, \cdots,-\hat{Q}\} .
\end{aligned}
$$

Furthermore, if the aforementioned inequality is feasible, the desired fault detection filters can be determined by

$$
L=Q_{1}^{-1} \vec{L}, V=\vec{V}
$$

Remark 4: In this paper, we first establish a comprehensive model that covers multi-rate sampled-data dynamics, network-induced fading measurements and randomly occurring faults, thereby better reflecting the reality of NCSs. In this case, sufficient conditions are given in Theorem 1-2 which make sure that the augmented system (8) is exponentially mean-square stable and $H_{\infty}$ criterion in (9) is satisfied. Note that, at this stage, the designed fault detection filter which shows the combined effects of fading parameters, fault occurrence probability as well as multi-rate multiple. Next, as the special case of networked multirate systems, i.e. $b=1$, the general networked single-rate systems with fading measurements and randomly occurring faults is taken into account, and corresponding fault detection filter is also designed in Corollary 1.

\section{TWO ILLUSTRATIVE EXAMPLES}

In this section, two numerical examples are presented to demonstrate the effectiveness of the proposed fault detection filter design scheme with fading measurements and randomly occurring faults for NMSs and NSSs, respectively.

Example 1 In this numerical example, for MSSs, the system parameters of (1) and (2) are chosen as follows:

$$
A=\left[\begin{array}{cc}
0.8 & h \\
0 & 0.6
\end{array}\right], B_{1}=\left[\begin{array}{c}
\frac{h^{2}}{2} \\
h
\end{array}\right], B_{2}=\left[\begin{array}{c}
\frac{3 h}{2} \\
0.6
\end{array}\right], C=\left[\begin{array}{ll}
0 & 0.3
\end{array}\right] .
$$

Here, the sampling period $h$ of system (1) is $0.5 s$, the measurement updating period is $1.5 s$ (i.e. $b=3$ ), the number of paths is $\ell=1$, the probability of the randomly occurring faults is $\bar{\alpha}=0.6$, and the probability density functions of channel coefficients are

$$
\left\{\begin{array}{l}
q\left(\beta_{0}\right)=0.0005\left(\mathrm{e}^{9.89 \beta_{0}}-1\right), \\
q\left(\beta_{1}\right)= \begin{cases}10 \beta_{1}, & 0 \leq \beta_{0} \leq 1 \leq 0.20 \\
-2.50\left(\beta_{1}-1\right), & 0.20<\beta_{1} \leq 1 ;\end{cases}
\end{array}\right.
$$

The mathematical expectations $\bar{\beta}_{s}$ can be calculated as 0.8991 and 0.4000 , and the variances $\tilde{\bar{\beta}}_{s}^{2}(s=0,1)$ are 0.0133 and 0.0467, respectively. By using the MATLAB LMI toolbox, for the augmented system (8), we obtain the minimum disturbance attenuation level as $\gamma_{*}=1.0094$. The sub-optimal FD filter can then be obtained as following:

$$
L=\left[\begin{array}{c}
2.1427 \\
-1.0263
\end{array}\right] ; V=-0.0389
$$




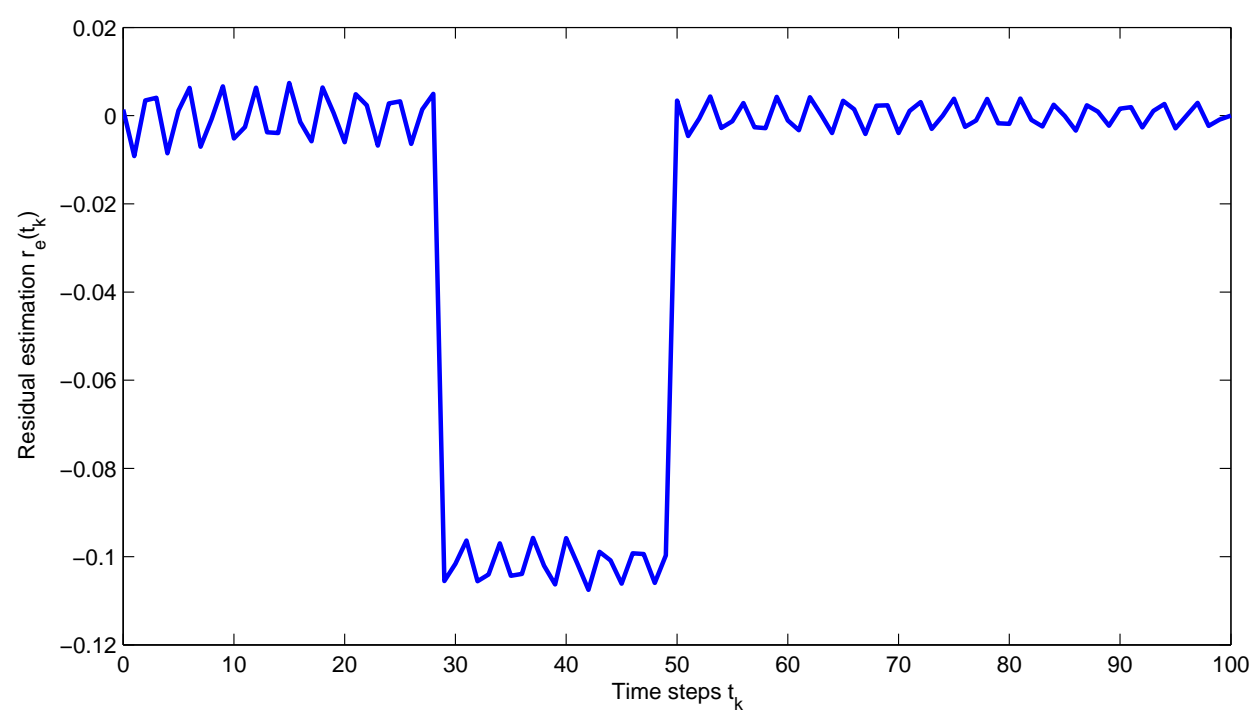

Fig. 2. Residual signal $r\left(t_{k}\right)$ for NMSs.

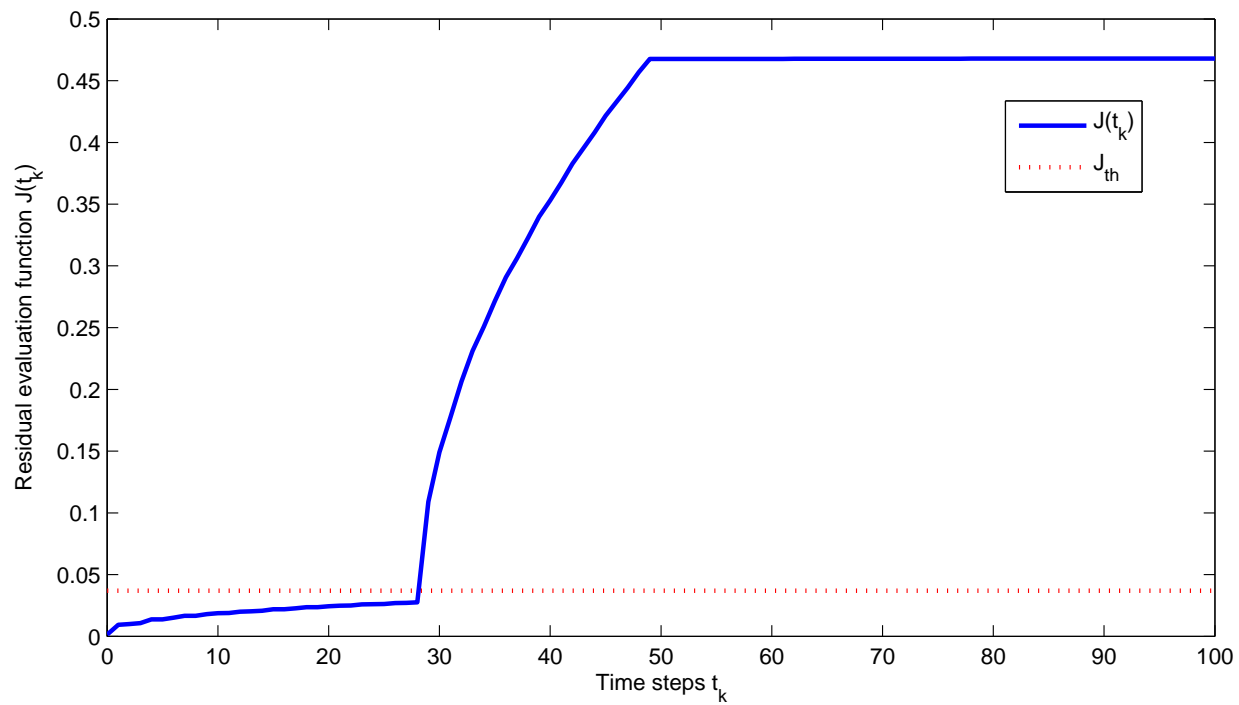

Fig. 3. Evolution of residual evaluation function $J\left(t_{k}\right)$ for NMSs.

Letting the initial state of (1) be $x\left(T_{0}\right)=\operatorname{col}\{0.1,-0.1\}$ and its estimation be $\hat{x}\left(t_{0}\right)=\operatorname{col}\{0.1,0\}$. To further illustrate the effectiveness of the designed fault detection filter, for $t_{k}=0,1,2, \cdots, 100$, let the fault signal and the disturbance input be given as

$$
f\left(t_{k}\right)=\left\{\begin{array}{cc}
0.1, & 30 \leq t_{k} \leq 50 \\
0, & \text { else }
\end{array}, \quad \omega\left(t_{k}\right)=e^{-0.01 t_{k}} \sin \left(2 t_{k}\right) .\right.
$$

The residual response $r\left(t_{k}\right)$ and evolution of residual evaluation function $J\left(t_{k}\right)=\left\{\sum_{h=t_{k_{0}}}^{t_{k}} r^{T}(h) r(h)\right\}^{\frac{1}{2}}$ for NMSs are shown in Figs. 2-3, respectively. After 200 runs of the simulations, we get an average value of $J_{t h}=0.0369$. From Fig. 3, it can be shown that $0.0275=J(29)<J_{t h}<J(30)=0.1090$, which means that the fault can be detected as soon as its occurrence.

We now examine the relationship between the disturbance attenuation level $\gamma$ and the fault occurrence probability $\bar{\alpha}$ as well as the multiple $b$ of the sampling period $h$. It can be observed from Table I that the 
disturbance attenuation performance deteriorates with increased $\bar{\alpha}$ and $b$, which is in agreement with the engineering practice.

TABLE I

THE PERMITTED MINIMUM $\gamma_{*}$.

\begin{tabular}{l|cccc}
\hline & $\bar{\alpha}=0.2$ & $\bar{\alpha}=0.4$ & $\bar{\alpha}=0.7$ & $\bar{\alpha}=0.9$ \\
\hline$b=2$ & 1.0008 & 1.0022 & 1.0035 & 1.0047 \\
$b=3$ & 1.0021 & 1.0057 & 1.0115 & 1.0145 \\
$b=4$ & 1.0109 & 1.0131 & 1.0145 & 1.0197 \\
\hline
\end{tabular}

Example 2 As the special case of NMSs, in this example, an internet-based three-tank system is introduced to illustrate the effectiveness of our proposed NSSs. With the variables defined in [32], the system model (1) and (2) with following parameters are adopted:

$$
A=\left[\begin{array}{ccc}
0.9974 & 0 & 0.0026 \\
0 & 0.9951 & 0.0024 \\
0.0026 & 0.0024 & 0.9950
\end{array}\right], B_{1}=\left[\begin{array}{cc}
16.2190 & 0 \\
0 & 16.2007 \\
0.0212 & 0.0193
\end{array}\right], B_{2}=\left[\begin{array}{c}
0.0212 \\
0.0193 \\
16.1997
\end{array}\right], C=\left[\begin{array}{lll}
1 & 0 & 0 \\
0 & 1 & 0
\end{array}\right] \text {. }
$$

where $x\left(T_{k}\right) \in R^{3}$ is the system state representing the liquid levels of the three tanks; similar to [32], $\omega\left(T_{k}\right) \in R^{2}$ is the disturbance used to model the unknown disturbance and input, $f\left(T_{k}\right) \in R$ is the fault signal reflecting the leakages in tank $3, y\left(T_{k}\right) \in R^{2}$ is the measurement output describing the height measurements of tank 1 and tank 2. Here, we mainly investigate the internet-based fault detection problem, the measurement signal will obtain through remote network, thus, due to the multi-path transmission and shadowing problem, network-induced channel fading and randomly occurring fault usually take place, then the actual received measurement signal through network is $\bar{y}\left(T_{k}\right) \in R^{2}$, which satisfies (3).

Our aim here is to detect the faults by using the established mathematic model of the system (1) as well as the measurement signals (2) through network in the presence of a leakage in tank 3 . In order to discuss simply the fault detection problem with fading measurement, we choose the fading parameters as (24). Choosing the faults occurrence probability as $\bar{\alpha}=0.6$, similar to Example 1, by using Corollary 1, the sub-optimal fault detection filter and the minimum $H_{\infty}$ attenuation level can be obtained as follows:

$$
L=\left[\begin{array}{ll}
-0.0042 & 0.0100 \\
-0.0030 & 0.0069 \\
-0.0095 & 0.0212
\end{array}\right], \quad V=10^{-5} \times\left[\begin{array}{ll}
0.1185 & -0.2856
\end{array}\right], \quad \gamma_{*}=1.0023 .
$$

The initial value of $(21)$ is chosen as $\bar{e}\left(T_{0}\right)=\operatorname{col}\{0.1,-0.1,0,0.2,0,-0.6,0,0.3,0\}$, for $T_{k}=$ $0,1,2, \cdots, 100$, the fault signal and exogenous disturbance input signal are set as

$$
f\left(T_{k}\right)=\left\{\begin{array}{cc}
0.5, & 30 \leq T_{k} \leq 50 \\
0, & \text { else }
\end{array}, \quad \omega\left(T_{k}\right)=\left[\begin{array}{c}
e^{-0.02 T_{k}} \sin \left(0.2 T_{k}\right) \\
e^{-0.01 T_{k}} \cos \left(0.1 T_{k}\right)
\end{array}\right] .\right.
$$

The residual response $r\left(T_{k}\right)$ and evolution of residual evaluation function $J\left(T_{k}\right)=\left\{\sum_{h=T_{0}}^{T_{k}} r^{T}(h) r(h)\right\}^{\frac{1}{2}}$ for NSSs are shown in Figs. 4-5, respectively. After 200 runs of the simulations, we get an average value of $J_{t h}=1.4582 \times 10^{-4}$. From Fig. 5, it can be shown that $1.3526 \times 10^{-4}=J(41)<J_{t h}<J(42)=$ $1.6304 \times 10^{-4}$, which means that the fault can be detected within 11 time steps after the fault occurred at $T_{k}=30$. 


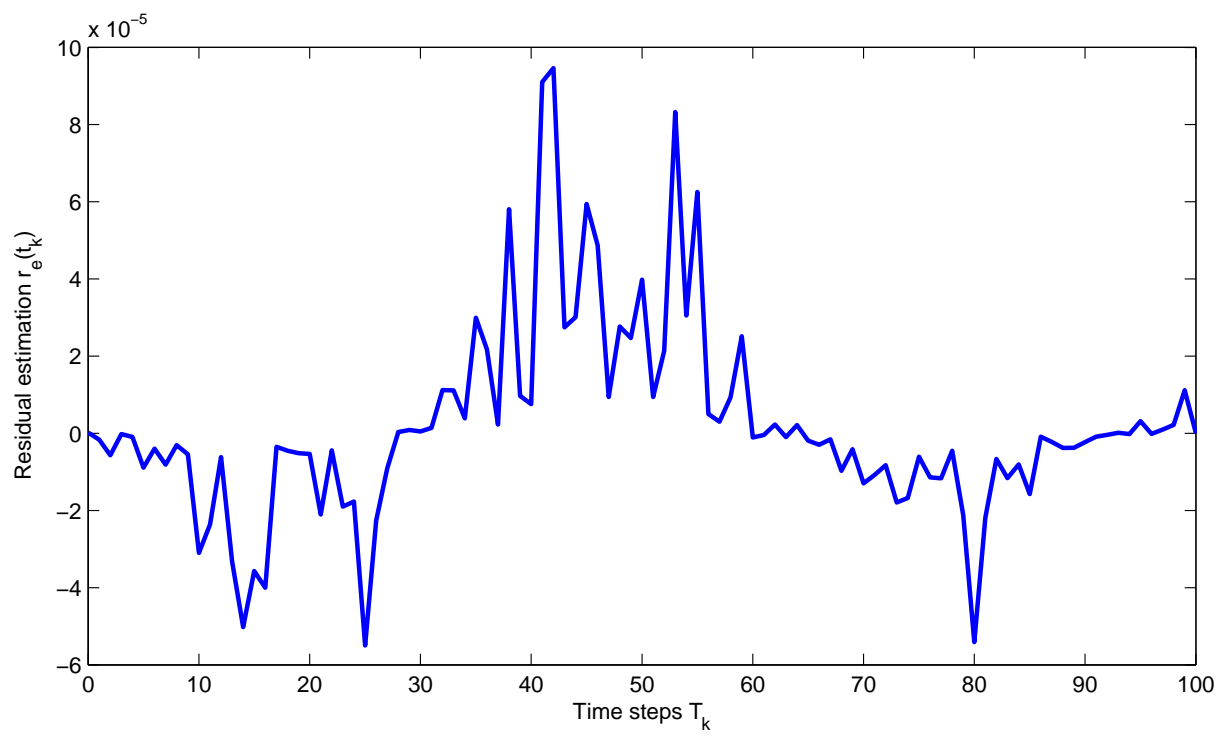

Fig. 4. Residual signal $r\left(T_{k}\right)$ for NSSs.

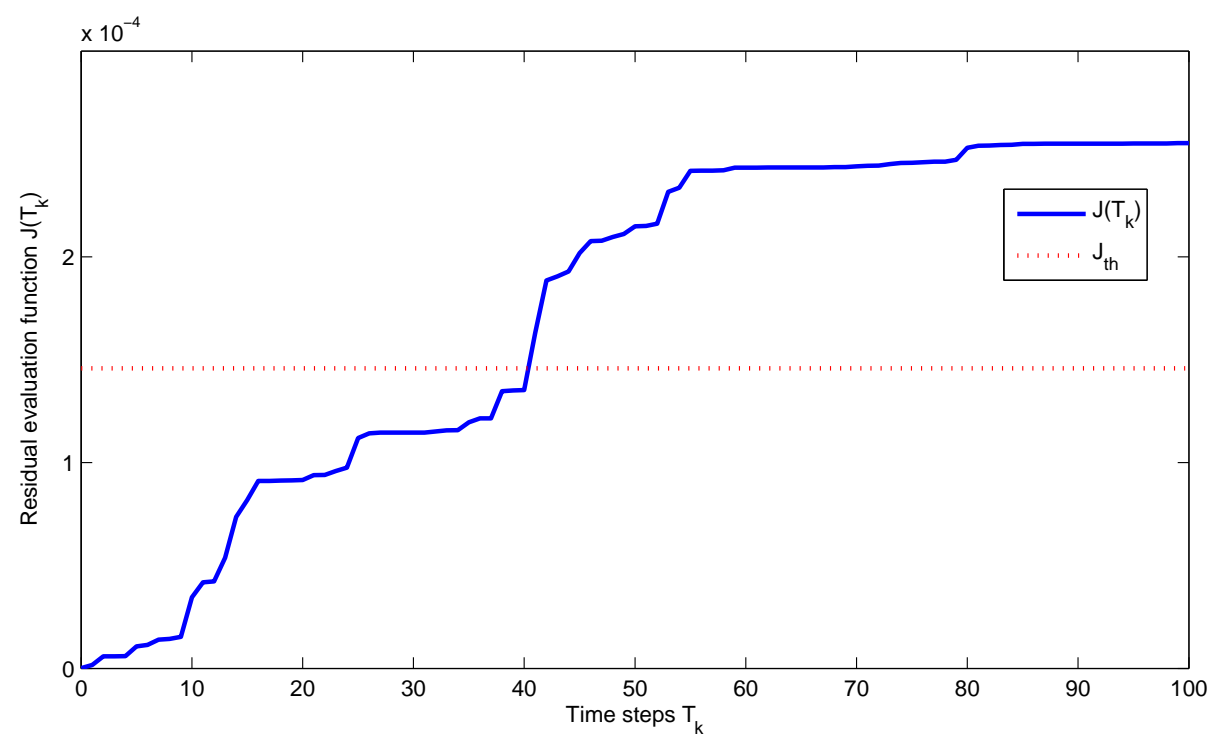

Fig. 5. Evolution of residual evaluation function $J\left(T_{k}\right)$ for NSSs.

\section{CONCLUSION}

In this paper, we have dealt with the fault detection problem for networked multi-rate systems with randomly occurring faults and fading measurements. Different from the existing results of fault detection for multi-rate sampled-data system, the delayed networked multi-rate systems is considered. By choosing linear matrix inequality technique and convex optimization tool so that we can use Matlab LMI Toolbox to design the fault detection filter effectively. Furthermore, as the special of NMSs, we also supply the result of fault detection for NSSs with randomly occurring faults and fading measurements. Two examples have been used to highlight the effectiveness of the proposed fault detection technology in this paper. It would be interesting to deal with the following future research topics [33]-[39]: 1) investigation on the 
impact from quantization strategies and event-triggered communication mechanism; and 2) extension of the techniques developed in this paper to more general time-varying and nonlinear systems.

\section{REFERENCES}

[1] M. Basin, S. Elvira-Ceja and E. Sanchez. Mean-square $H_{\infty}$ filtering for stochastic systems: application to a 2DOF helicopter. Signal Processing, vol. 92, no. 3, pp. 801-806, 2012.

[2] R. Caballero-Águila, A. Hermoso-Carazo and J. Linares-Pérez. Optimal state estimation for networked systems with random parameter matrices, correlated noises and delayed measurements. International Journal of General Systems vol. 44, no. 2, pp. 142-154, 2015.

[3] H. Gao, T. Chen and J. Lam, A new delay system approach to network-based control, Automatica, Vol. 44, No. 1, pp. 39-52, 2008.

[4] Z. Du, D. Yue and S. Hu, $H_{\infty}$ stabilization for singular networked cascade control systems with state delay and disturbance, IEEE Transactions on Industrial Informatics, Vol. 10, No. 2, pp. 882-894, 2014.

[5] Z. Wang, F. Yang, D.W.C. Ho and X. Liu, Robust $H_{\infty}$ control for networked systems with random packet losses, IEEE Transactions on Systems, Man, and Cybernetics - Part B, Vol. 37, No. 4, pp. 916-924, 2007.

[6] J. Xiong and J. Lam, Stabilization of linear systems over networks with bounded packet loss, Automatica, Vol. 43, No. 1, pp. 80-87, 2007.

[7] J. Hu, J. Liang, D. Chen, D. Ji and J. Du, A recursive approach to non-fragile filtering for networked systems with stochastic uncertainties and incomplete measurements, The Journal of The Franklin Institute, Vol. 352, No. 5, pp. 1946-1962, 2015.

[8] G. Wei, S. Liu, Y. Song and Y. Liu, Probability-guaranteed set-membership filtering for systems with incomplete measurements, Automatica, Vol. 60, pp. 12-16, 2015.

[9] E. Tian, D. Yue and X. Zhao, Quantised control design for networked control systems, IET Control Theory and Applications, Vol. 1, No. 6, pp. 1693-1699, 2007.

[10] Y. Zou, J. Lam, Y. Niu and D. Li, Constrained predictive control synthesis for quantized systems with Markovian data loss, Automatica, Vol. 55, pp. 217-225, 2015.

[11] H. Dong, Z. Wang, S. X. Ding and H. Gao, Finite-horizon reliable control with randomly occurring uncertainties and nonlinearities subject to output quantization, Automatica, Vol. 52, pp. 355-362, 2015.

[12] N. Elia, Remote stabilization over fading channels, Systems and Control Letters, No. 3, pp. 237-249, 2005.

[13] A.S. Leong, S. Dey, G. Nair and P. Sharma, Power allocation for outage minimization in state estimation over fading channels, IEEE Transactions on Signal Processing, Vol. 59, No. 7, pp. 3382-3397, 2011.

[14] N. Xiao, L. Xie and L. Qiu, Feedback stabilization of discrete-time networked systems over fading channels, IEEE Transactions on Automatic Control, Vol. 57, No. 9, pp. 2176-2189, 2012.

[15] D. Ding, Z. Wang, B. Shen and H. Dong, $H_{\infty}$ state estimation with fading measurements, randomly varying nonlinearities and probabilistic distributed delays, International Journal of Robust and Nonlinear Control, Vol. 25, No. 13, pp. $2180-2195,2015$.

[16] S. Zhang, Z. Wang, D. Ding, H. Dong, F. E. Alsaadi and T. Hayat, Non-fragile $H_{\infty}$ fuzzy filtering with randomly occurring gain variations and channel fadings, IEEE Transactions on Fuzzy Systems, in press, DOI: 10.1109/TFUZZ.2015.2446509.

[17] H. Dong, Z. Wang, S. X. Ding and H. Gao, Event-based $H_{\infty}$ filter design for a class of nonlinear time-varying systems with fading channels and multiplicative noises, IEEE Transactions on Signal Processing, Vol. 63, No. 13, pp. 3387-3395, 2015.

[18] M. Fadali, Observer-based robust fault detection of multi-rate linear system using a lift reformulation, Computers and Electrical Engineering, Vol. 29, No. 1, pp. 235-243, 2003.

[19] I. Izadi, Q. Zhao and T. Chen, An optimal scheme for fast rate fault detection based on multi-rate sampled data, Journal of Process Control, Vol. 15, No. 3, pp. 307-319, 2005.

[20] M. Zhong, H. Ye, S.X. Ding and G. Wang, Observer-based fast rate fault detection for a class of multi-rate sampled-data systems, IEEE Transactions on Automatic Control, Vol. 52, No. 3, pp. 520-525, 2007.

[21] Y. Liang, T. Chen and Q. Pan, Multi-rate stochastic $H_{\infty}$ filtering for networked multi-sensor fusion, Automatica, Vol. 46, No. 2, pp. 437-444, 2010.

[22] W.-A. Zhang, G. Feng and L. Yu, Multi-rate distributed fusion estimation for sensor networks with packet losses, Automatica, Vol. 48, No. 9, pp. 2016-2028, 2012.

[23] J. Gertler, Fault Detection and Diagnosis in Engineering Systems, Dekker, New York,1998.

[24] S. Ding, Model-based Fault Diagnosis Techniques: Design Schemes, Algorithms, Tools, Berlin, Germany: Springer-Verlag, 2008.

[25] H. Fang, H. Ye and M. Zhong, Fault diagnosis of networked control systems, Annual Reviews in Control, Vol. 31, No. 1, pp. 55-68, 2007.

[26] Y. Long and G.-H. Yang, Fault detection for a class of networked control systems with finite-frequency servo inputs and random packet dropouts, IET Control Theory and Applications, Vol. 6, No. 15, pp. 2397-2408, 2012.

[27] J. Feng, S. Wang and Q. Zhao, Closed-loop design of fault detection for networked non-linear systems with mixed delays and packet losses, IET Control Theory and Applications, Vol. 7, No. 6, pp. 858-868, 2013. 
[28] H. Dong, Z. Wang, S. X. Ding and H. Gao, Finite-horizon estimation of randomly occurring faults for a class of nonlinear time-varying systems, Automatica, Vol. 50, No. 12, pp. 3182-3189, 2014.

[29] Y. Zhang, Z. Liu, H. Fang and H. Chen, $H_{\infty}$ fault detection for nonlinear networked systems with multiple channels data transmission pattern, Information Sciences, Vol. 221, No. 1, pp. 534-543, 2013.

[30] Y. Zheng, H. Fang and H. Wang, Takagi-sugeno fuzzy-model-based fault detection for networked control systems with Markov delays, IEEE Transactions on Systems, Man, and Cybernetics, Part B: Cybernetics, Vol. 36, No. 4, pp. 924-929, 2006.

[31] Z. Mao, B. Jiang and P. Shi, Protocol and fault detection design for nonlinear networked control systems, IEEE Transactions on Circuits and Systems-II: Express Briefs, Vol. 56, No. 3, pp. 255-259, 2009.

[32] X. He, Z. Wang, Y. Ji and D.H. Zhou, Robust fault detection for networked systems with distributed sensors, IEEE Transactions on Aerospace and Electronic Systems, Vol. 47, No. 1, pp. 166-177, 2011.

[33] X. Wang, E. E. Yaz and J. Long, Robust and resilient state-dependent control of continuous-time nonlinear systems with general performance criteria, Systems Science and Control Engineering: An Open Access Journal, Vol. 2, No. 1, pp.34-40, 2014.

[34] D. Ding, Z. Wang, B. Shen and H. Dong, Envelope-constrained $H_{\infty}$ filtering with fading measurements and randomly occurring nonlinearities: the finite horizon case, Automatica, Vol. 55, pp. 37-45, 2015.

[35] M. Chen, W. Chen and Q. Wu, Adaptive fuzzy tracking control for a class of uncertain MIMO nonlinear systems using disturbance observer, Science China: Information Sciences, Vol. 57, No. 1, pp. 1-13, 2014.

[36] L. Sheng, W. Zhang and M. Gao, Relationship between Nash equilibrium strategies and $H_{\infty} / H_{2}$ control of stochastic Markov jump systems with multiplicative noise, IEEE Transactions on Automatic Control, Vol. 59, No. 9, pp. 2592-2597, 2014.

[37] M. Lefebvre and F. Zitouni, Analytical solutions to LQG homing problems in one dimention, Systems Science and Control Engineering: An Open Access Journal, Vol. 2, No. 1, pp. 41-47, 2014.

[38] X. Lan, Y. Wang and L. Liu, Dynamic decoupling tracking control for the polytopic LPV model of hypersonic vehicle, Science China: Information Sciences, Vol. 58, No. 9, pp. 1-14, 2015.

[39] D. Ding, Z. Wang, J. Lam and B. Shen, Finite-Horizon $H_{\infty}$ control for discrete time-varying systems with randomly occurring nonlinearities and fading measurements, IEEE Transactions on Automatic Control, in press, DOI:10.1109/TAC.2014.2380671. 